\title{
A practical algorithm for the computation of tree felling times
}

\author{
D. S. Holloway ${ }^{1}$
}

(Received 2 August 2007; revised 1 April 2008)

\begin{abstract}
The time taken for a tree to fall when felled can be expressed in closed form in terms of elliptic integrals. However, this form of the solution is of limited practical use to the non-mathematician. A straightforward numerical method is presented that can be programmed into a spreadsheet in a few lines without requiring any high level programming or mathematical skills. Good accuracy for practical purposes is obtained without any iteration, but accuracy is further improved with two or three iterations, each involving only one or two extra cells in the spreadsheet. Results are compared with the analytic solution. This article demonstrates how a challenging real problem is accurately solved numerically with basic office tools and limited mathematics.
\end{abstract}

See http://anziamj.austms.org.au/ojs/index.php/ANZIAMJ/article/view/368 for this article, (c) Austral. Mathematical Soc. 2008. Published April 15, 2008. ISSN 1446-8735 


\section{Contents}

1 Introduction

C527

2 Problem definition

C528

3 Analytic solution

C530

4 Numerical solution

C531

4.1 Fourth order approximation . . . . . . . . . . .

C533

4.2 Sixth order approximation . . . . . . . . . . .

C534

5 Results

C535

6 Conclusion

C539

References

C539

\section{Introduction}

The subject of this article is depicted by the free body diagram in Figure 1. When a tree is felled its motion to the ground occurs in two stages: the initial rotation about its point of contact with the stump; followed by free fall after separation from the stump with constant vertical acceleration and constant angular velocity. This article considers only the first stage, which cannot be described in terms of elementary functions. The second is trivially described mathematically, though is complicated in practice by the need to determine which part of the tree strikes the ground first.

In order to render the problem soluble I assume

- only gravitational forces and contact forces at the stump are significant, 


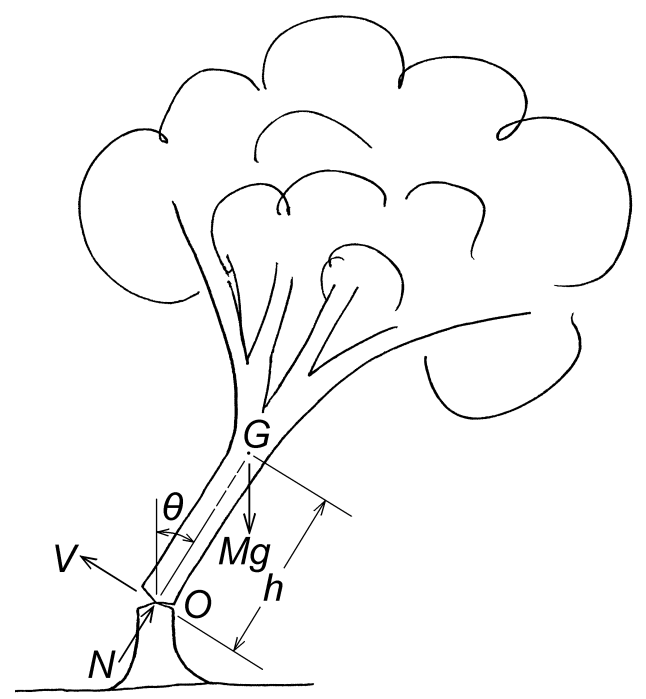

FiguRE 1: Schematic diagram of tree being felled showing relevant algebraic variables

- there is no moment at the point of contact with the stump,

- aerodynamic drag is negligible,

- adequate friction exists at the stump to restrain translation before separation (the cut is jagged), and

- there is no interference to the motion, for example the tree canopy does not contact the ground before separation.

\section{Problem definition}

In Figure 1, valid until the instant of separation, $O$ is the point of contact between the tree and its stump about which the centre of mass $G$ describes 
circular motion with radius $h$. The tree and the cut are not naturally symmetric so points $O$ and $G$ may be located anywhere with respect to the trunk cross section, but $\theta$ is the angle that $O G$ makes with the vertical and is assumed initially to have a finite positive value $\alpha$. Under the action of gravity $\theta$ must increase with time. A contact force exists at $O$ represented by components $N$ and $V$ respectively perpendicular and parallel to $O G$, and kinematics of circular motion gives the components of the acceleration of $G$ as $h \ddot{\theta}$ and $h \dot{\theta}^{2}$ also respectively perpendicular and parallel to $O G$. These directions are therefore the natural choice in which to resolve force components when writing the equations of motion. A gravitational force $M g$ acts vertically at $G$. The portion of the tree above the cut has mass $M$ and moment of inertia about point $O$ of $I_{O}$. Newton's equations of dynamic equilibrium are described in any university introductory engineering dynamics text such as that by Meriam and Kraige [2], and applied to this problem are

$$
\begin{aligned}
I_{O} \ddot{\theta} & =M g h \sin \theta, \\
M h \ddot{\theta} & =M g \sin \theta-V, \\
M h \dot{\theta}^{2} & =M g \cos \theta-N,
\end{aligned}
$$

with initial conditions

$$
\begin{aligned}
& \theta(0)=\alpha, \\
& \dot{\theta}(0)=0 .
\end{aligned}
$$

We determine implicitly the time to separation by seeking as follows the time variation of the trunk angle $\theta(t)$ and the angle $\theta=\beta$ at which the tree separates from the stump.

Multiplying (1) by $\dot{\theta}$ and defining the constant

$$
A=\sqrt{\frac{I_{O}}{M g h}}
$$


yields

$$
A^{2} \dot{\theta} \ddot{\theta}=\dot{\theta} \sin \theta .
$$

Then integrate (7) with respect to time with initial conditions (4) and (5),

$$
\frac{1}{2} A^{2} \dot{\theta}^{2}=\cos \alpha-\cos \theta
$$

Substitute the value of $\dot{\theta}^{2}$ thus obtained into the axial equilibrium equation (3) at the instant of separation of the tree from the stump, at which $N=$ 0 , to give the angle of separation as

$$
\beta=\arccos \left\{\frac{\cos \alpha}{1+I_{O} / 2 M h^{2}}\right\} .
$$

I note that since $\cos \alpha<1, I_{O}=I_{G}+M h^{2}$ and $I_{G}$, the mass moment of inertia about the centre of gravity $G$, is always positive then $\beta>\arccos 2 / 3 \simeq$ $48.2^{\circ}$. This is an absolute lower bound. More realistic bounds (33) and (34) are discussed in Section 5 .

The remaining task is to solve equation (8) between the limits $\theta(0)=\alpha$ and $\theta(t)=\beta$, hence determine $t$.

\section{Analytic solution}

I solve differential equation (8) by separation of variables,

$$
\frac{A}{\sqrt{2}} \int_{\alpha}^{\beta} \frac{d \theta}{\sqrt{\cos \alpha-\cos \theta}}=\int_{0}^{t} d t=t .
$$

Now with the change of variable $\theta=\pi-2 \phi$ and defining $\phi_{0}=(\pi-\alpha) / 2=\phi$ at $t=0$,

$$
t=\frac{A}{\sqrt{2}} \int_{\frac{\pi-\alpha}{2}}^{\frac{\pi-\beta}{2}} \frac{-2 d \phi}{\sqrt{2} \sqrt{\sin ^{2} \phi_{0}-\sin ^{2} \phi}}
$$




$$
\begin{aligned}
& =\frac{A}{\sin \phi_{0}}\left\{\int_{0}^{\frac{\pi-\alpha}{2}} \frac{d \phi}{\sqrt{1-m \sin ^{2} \phi}}-\int_{0}^{\frac{\pi-\beta}{2}} \frac{d \phi}{\sqrt{1-m \sin ^{2} \phi}}\right\} \\
& =\frac{A}{\sin \phi_{0}}\left\{F\left(\frac{\pi-\alpha}{2} \mid m\right)-F\left(\frac{\pi-\beta}{2} \mid m\right)\right\},
\end{aligned}
$$

where $m=1 / \sin ^{2} \phi_{0}$. Here $t$ is expressed in terms of elliptic integrals of the first kind $F(\varphi \mid m)$ defined by Abramowitz and Stegun [1, $\S 17.2]$.

It is usual to transform $F$ so that $0 \leq m \leq 1$. I use the result $F(\varphi \mid m)=$ $m^{-1 / 2} F\left(\theta \mid m^{-1}\right)$ with $\sin \theta=m^{1 / 2} \sin \varphi[1, \S 17.4 .15]$. After some simplification,

$$
\begin{aligned}
t & =A\left\{F\left(\frac{\pi}{2} \mid \cos ^{2} \frac{\alpha}{2}\right)-F\left(q_{\beta} \mid \cos ^{2} \frac{\alpha}{2}\right)\right\} \\
& =A\left\{K\left(\cos ^{2} \frac{\alpha}{2}\right)-F\left(q_{\beta} \mid \cos ^{2} \frac{\alpha}{2}\right)\right\},
\end{aligned}
$$

where $q_{\beta}=\arcsin \left(\frac{\cos \beta / 2}{\cos \alpha / 2}\right), A$ is given by (6) and $K$ is the complete elliptic integrals of the first kind [1, S17.3].

Further reduction using double angle formulas and the expression (9) for $\beta$ gives

$$
q_{\beta}=\arcsin \sqrt{\frac{1+\cos \alpha /\left(1+\frac{I_{O}}{2 M h^{2}}\right)}{1+\cos \alpha}} .
$$

Thus the time to separation, normalised by $A$, is a function of $I_{O} / 2 M h^{2}$ and $\alpha$ only.

\section{Numerical solution}

The analytic solution may be concisely coded in programs such as Matlab. However, this work was undertaken for a client from a background without 
high level mathematical skills and with access only to spreadsheets to perform computations. A numerical method was sought that

- was practical and easily used by non-mathematicians,

- was easily implemented in a spreadsheet, and

- gave appropriate accuracy bearing in mind the imprecise nature of the input parameters.

Errors of less than $2 \%$ were deemed to meet or exceed the last criterion.

Traditional time stepping methods used for ODEs were considered unsuitable because they generally require a large number of time steps to achieve reasonable accuracy, which would quickly clutter a spreadsheet, and they would require interpolation or iteration as they do not in general explicitly solve for $t$.

In contrast a method based on a truncated Taylor series proved ideal.

First, expand $\theta(t)=\beta$ as a Taylor series where $t$ is time to separation,

$$
\beta=\theta(0)+\dot{\theta}(0) t+\ddot{\theta}(0) \frac{t^{2}}{2}+\cdots .
$$

We have from (1) and (6) that $\ddot{\theta}=\sin \theta / A^{2}$, giving sufficient information to express arbitrarily high order derivatives of $\theta$ in terms of $\theta$ and $\dot{\theta}$. Up to sixth order,

$$
\begin{aligned}
& A^{2} \frac{d^{3} \theta}{d t^{3}}=\cos \theta \dot{\theta} \\
& A^{2} \frac{d^{4} \theta}{d t^{4}}=\ddot{\theta} \cos \theta-\dot{\theta}^{2} \sin \theta \\
& A^{2} \frac{d^{5} \theta}{d t^{5}}=\frac{d^{3} \theta}{d t^{3}} \cos \theta-3 \ddot{\theta} \dot{\theta} \sin \theta-\dot{\theta}^{3} \cos \theta
\end{aligned}
$$




$$
\begin{aligned}
A^{2} \frac{d^{6} \theta}{d t^{6}}= & \frac{d^{4} \theta}{d t^{4}} \cos \theta-4 \frac{d^{3} \theta}{d t^{3}} \dot{\theta} \sin \theta \\
& -6 \ddot{\theta} \dot{\theta}^{2} \cos \theta-3 \ddot{\theta}^{2} \sin \theta+\dot{\theta}^{4} \sin \theta .
\end{aligned}
$$

Putting $\dot{\theta}(0)=0$ and $\theta(0)=\alpha$ reduces even derivatives at $t=0$ to

$$
\begin{aligned}
\frac{d^{4} \theta(0)}{d t^{4}} & =\frac{1}{A^{4}} \sin \alpha \cos \alpha, \\
\frac{d^{6} \theta(0)}{d t^{6}} & =\frac{1}{A^{6}} \sin \alpha\left(\cos ^{2} \alpha-3 \sin ^{2} \alpha\right),
\end{aligned}
$$

and odd derivatives vanish. Thus to sixth (or seventh) order

$$
\beta=\alpha+\sin \alpha \frac{\tau^{2}}{2 !}+\sin \alpha \cos \alpha \frac{\tau^{4}}{4 !}+\sin \alpha\left(\cos ^{2} \alpha-3 \sin ^{2} \alpha\right) \frac{\tau^{6}}{6 !},
$$

where I introduce dimensionless time $\tau=t / A$.

If it is reasonable to assume that the tree does not lean substantially, then the small $\alpha$ approximation may be used:

$$
\beta \simeq \alpha\left\{1+\frac{\tau^{2}}{2 !}+\frac{\tau^{4}}{4 !}+\left(1-3 \alpha^{2}\right) \frac{\tau^{6}}{6 !}\right\}
$$

\subsection{Fourth order approximation}

To fourth order the problem is described by the quadratic in $\tau^{2}$

$$
a \tau^{4}+b \tau^{2}+c=0,
$$

where

$$
\begin{aligned}
& a=\frac{\sin \alpha \cos \alpha}{4 !}, \\
& b=\frac{\sin \alpha}{2 !},
\end{aligned}
$$




$$
c=\alpha-\beta \text {. }
$$

The time to separation may be obtained explicitly as the solution to (23). Since $c<0$ one of the roots $\tau^{2}$ of (23) will be negative and not physically meaningful, so the positive root is to be taken. However, the results by this method differed from the analytic solution by up to $10 \%$ for typical input parameters, so a higher order approximation was deemed necessary.

\subsection{Sixth order approximation}

Comparing (21) and (23), sixth order accuracy is obtained by replacing $c$ in (26) with

$$
c_{i}=\alpha-\beta+\frac{\tau_{i}^{6}}{6 !} \sin \alpha\left(\cos ^{2} \alpha-3 \sin ^{2} \alpha\right) .
$$

Solving the quadratic in $\tau^{2}(23)$,

$$
\tau_{i+1}=\sqrt{\frac{6}{\cos \alpha}}\left(\sqrt{1-\frac{2 c_{i}}{3} \cot \alpha}-1\right)^{1 / 2} .
$$

In this case it is necessary to iterate between (27) and (28) to obtain successive approximations $\tau_{i}$. The initial approximation $\tau_{1}=0$ was used and 2-3 iterations were sufficient to confine errors to within $2 \%$, requiring only $4-6$ additional lines in the spreadsheet.

The sixth order small $\alpha$ approximation, after cancelling common factor $\alpha$, is

$$
\begin{aligned}
a & =\frac{1}{4 !}, \\
b & =\frac{1}{2 !}, \\
c & =1-\frac{\beta}{\alpha}+\frac{\tau^{6}}{6 !}\left(1-3 \alpha^{2}\right),
\end{aligned}
$$


from which

$$
\tau=\sqrt{6}\left(\sqrt{1-\frac{2 c}{3}}-1\right)^{1 / 2}
$$

Three sources of error are present in this numerical method arising from

1. truncation of the Taylor series - sixth order terms were necessary and in most cases sufficient to meet the accuracy criterion;

2. termination of the iteration between (31) and (32) - this error is easily controlled, but 2-3 iterations were found sufficient to meet the accuracy criterion, and underrelaxation by averaging the new and old $\tau$ accelerated convergence;

3. the small $\alpha$ approximation (if used) - this proved of no concern for $\alpha<$ $15^{\circ}$.

Error results presented in Figure 4 are discussed in more detail below.

\section{$5 \quad$ Results}

Figure 2 shows theoretical dimensionless felling times computed analytically by (12) for various initial and separation angles. Each value of $\beta$ implies a certain $I_{0} / M h^{2}$, not all of which are possible as noted in section 2 . To identify likely values of $\beta$ consider $I_{O} / M h^{2}$ in the denominator of (9) and recall that $I_{O}=I_{G}+M h^{2}$. A realistic but still conservative lower bound for $I_{G}$ is obtained by assuming the tree to approximate a uniform slender rod of length $2 h$, thus $I_{G} \geq M h^{2} / 3$ and from (9)

$$
\beta \geq \arccos \left(\frac{\cos \alpha}{5 / 3}\right) \geq 53.13^{\circ} .
$$




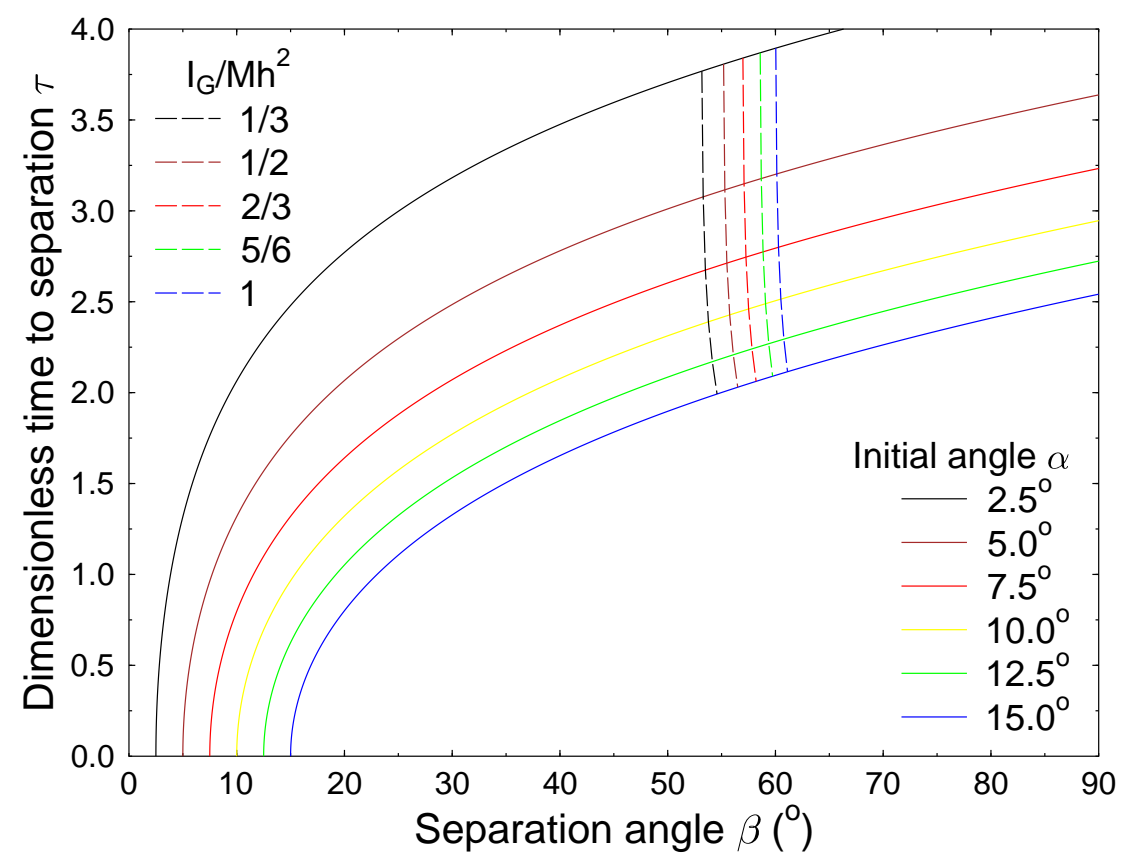

Figure 2: Felling time as a function of $\beta$ : Solid lines, constant $\alpha$; Dashed lines, constant $I_{G} / M h^{2}$. 


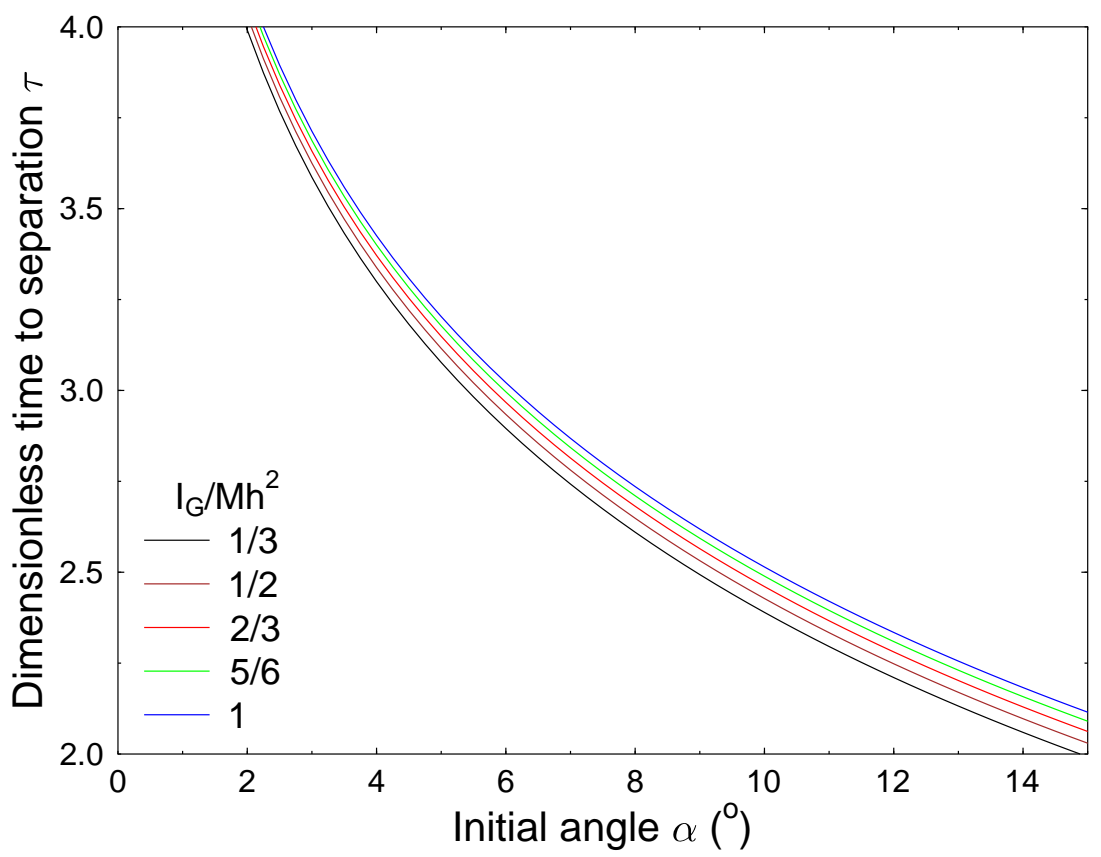

Figure 3: Felling times as a function of initial angle $\alpha$ for various $I_{G} / M h^{2}$.

If the tree is approximated as a uniform sphere of radius $H, I_{G}=\frac{2}{5} M h^{2}$ and $\beta \geq \arccos (\cos \alpha / 1.7)$. However, as a practical upper bound for $\beta$ I assume $\alpha=15^{\circ}$ and a denominator of 2 in (9), corresponding to $I_{G}=M h^{2}$, giving

$$
\beta \leq \arccos \left(\frac{\cos 15^{\circ}}{2}\right) \simeq 61.12^{\circ} .
$$

Values within these limits are shown in Figure 2 by the dashed lines, representing contours of constant $I_{G} / M h^{2}$ within the above range. Note that quite a narrow range of $\beta$ results.

Figure 3 shows felling time as a function of initial angle and $I_{G} / M h^{2}$, which together are sufficient to uniquely determine it. We see perhaps sur- 


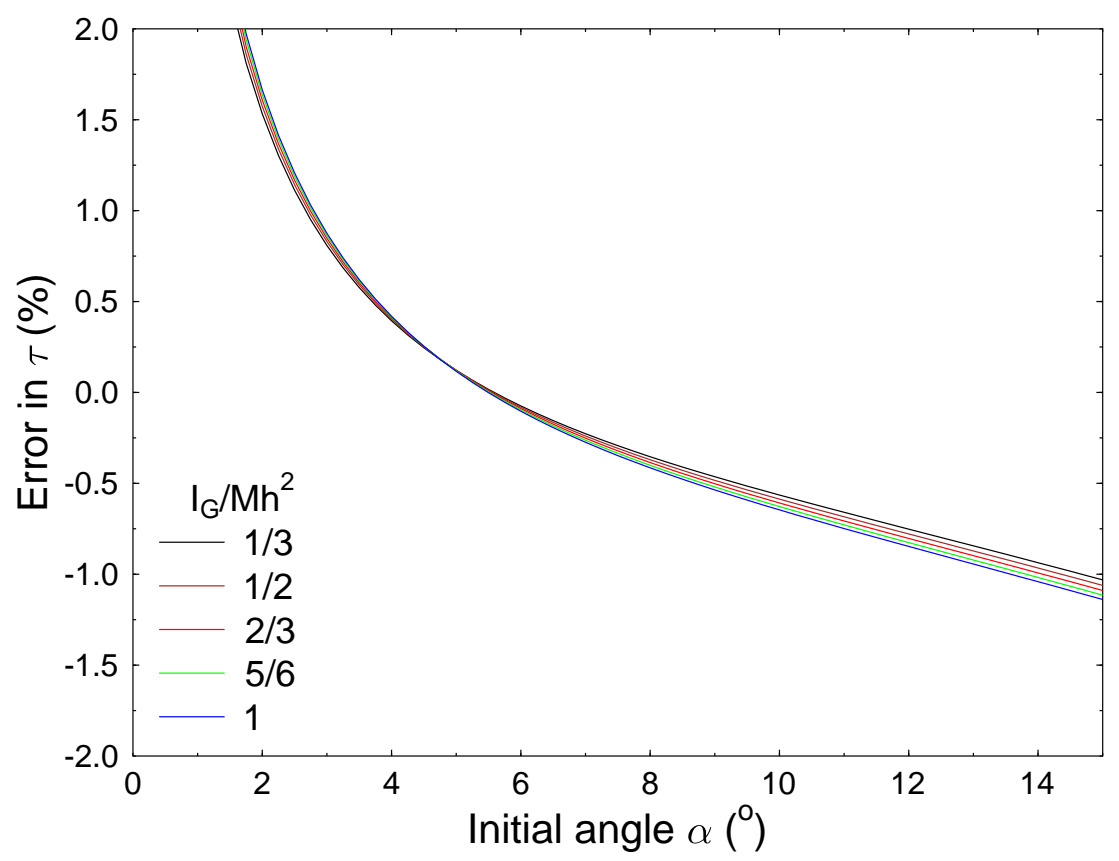

Figure 4: Error in computed felling times using the 6th order small $\alpha$ approximation.

prisingly that the tree's moment of inertia has relatively little influence on the felling time, while as expected the felling time is strongly dependent on the initial lean angle $\alpha$.

Figure 4 shows that error in the sixth order small $\alpha$ approximation is predominantly a function of $\alpha$. For small $\alpha, \tau$ increases rapidly and the error therefore is dominated by the truncation of the Taylor series. The error can be reduced if necessary by inclusion of higher order terms. For large $\alpha$ the error is dominated by the small $\alpha$ approximation. This component can be eliminated entirely by using the finite $\alpha$ form in which (27) and (28) replace (31) and (32). 


\section{Conclusion}

The numerical method of Section 4 proved a simple and accurate means to determine the time taken for a cut tree to fall to its point of separation from the stump. In this method the time

$$
t=A \tau\left(\alpha, I_{O} / M h^{2}\right)
$$

is fully specified by equations (6), (9), (31) and (32).

The method achieved the desired objectives that

- it does not require high level mathematical skills - it can be understood using first year undergraduate mathematics and implemented with much less;

- it is easily programmed in a spreadsheet;

- it introduces errors of less than $2 \%$ over a realistic range of inputs, which is considerable less than the uncertaintly in the input parameters.

\section{References}

[1] Milton Abramowitz and Irene A Stegun. Handbook of Mathematical Functions with Formulas, Graphs and Mathematical Tables, volume 55 of Applied Mathematics. National Bureau of Standards, 1964. C531

[2] J.L. Meriam and L.G. Kraige. Engineering Mechanics Volume 2 Dynamics. 2nd edition, 1998. C529 


\section{Author address}

1. D. S. Holloway, School of Engineering, University of Tasmania, Hobart, Australia.

mailto:Damien. Holloway@utas . edu . au 